\title{
New LS and SVD Based Methods for Estimating Frequencies of Complex Sinusoids
}

\author{
George V. Moustakides and Kostas Berberidis \\ Dept. of Computer Engineering, Univ. of Patras, GREECE. \\ Computer. Techn. Inst. (CTI), P.O. Box 1122, Patras 26110, GREECE.
}

\begin{abstract}
New LS and SVD based methods for the estimation of frequencies of complex sinusoids in white noise are presented. The methods are based on a new prediction problem that has some very useful properties leading to algorithms that have considerably reduced complexity. This is achieved without practically sacrificing any performance with respect to existing methods that are based on the Forward - Backward predictor.
\end{abstract}

\section{INTRODUCTION}

It is well established that fitting linear prediction models to processes consisting of complex sinusoids in noise results in high resolution spectral line estimation beyond the limits of traditional techniques. The associated linear predictor may be computed either by using LS spectral analysis techniques [1],[2] or using SVD based techniques $[2],[5]$ to reduce the effect of noise in the associated autocorrelation matrix. At medium to high SNR both techniques have comparable performance but at medium to low SNR the SVD based methods seem to outperform considerably their LS counterparts.

In the case of undamped exponentials in noise the problem leads naturally to the use of conjugate symmetric predictors. Until recently the only known symmetric predictor applicable to this problem was the symmetric Smoother [1],[3]. In [4] a new symmetric predictor, the Bidirectional, was introduced and shown to be suitable for spectral line estimation. This predictor was defined only for the real data case and there was no study of its performance.

In this paper, first, the LS complex bidirectional predictor is defined. The extension to the complex case is not straightforward and requires a solution to a double minimization problem. Asymptotic as well as extensive experiments show the superiority of the Bidirectional predictor with respect to Smoother. This performance is accompanied with a significant computational saving. Second, an SVD based technique for the symmetric prediction problem is developed. Two methods one for the Bidirectional and one for the Smoother are presented. Due to the rich structure of the bidirectional prediction problem the corresponding method performs almost identically as the modified Forward-Backward linear prediction method [5] but requiring much less computational burden

\section{THE COMPLEX BIDIRECTIONAL PREDICTOR}

Let us assume that we are given a complex data sequence $\{x(n)\}, n=1, \ldots, N$ consisting of $p$ undamped exponentials in white noise, i.e.

$$
x(n)=\sum_{k=1}^{p} A_{k} e^{j\left(2 \pi f_{k} n+\theta_{k}\right)}+w(n)=\sum_{k=1}^{p} h_{k} z_{k}^{n}+w(n)
$$

where $z_{k}=e^{j 2 \pi f_{k}}$ and $h_{k}=A_{k} e^{j \theta_{k}}$. Let us call $P_{p}(z)$ the polynomial of order $p$ that has as roots the $z_{k}, k=$ $1, \cdots, p$, that is

$$
P_{p}(z)=\prod_{k=1}^{p}\left(1-z^{-1} z_{k}\right)=\sum_{k=0}^{p} a_{k} z^{-k}
$$

Since the all roots of $P_{p}(z)$ lie on the unit circle the coefficients of the polynomial have some very characteristic properties [1]. Notice that the coefficient $a_{p}$ has unit modulus (as being the product of all $z_{k}$ ). Thus if $a_{p}=e^{j 2 \phi}$ and we define $G_{p}(z)=e^{-\phi} P_{p}(z)$ then it can be shown that the polynomial $G_{p}(z)$ has conjugate symmetric coefficients, namely, if $g_{p+1}$ is the vector of its coefficients then

$$
\boldsymbol{g}_{p+1}=\left[\begin{array}{llllll}
e^{-j \phi} g_{1} & g_{2} & \cdots & g_{2}^{*} & g_{1}^{*} & e^{j \phi}
\end{array}\right]^{t}=J g_{p+1}^{*}
$$

where $J$ is the so called exchange matrix having unities along the cross diagonal and zeros everywhere else, and “*” denotes complex conjugate. If we apply the filter $G_{p}(z)$ to noiseless data then we conclude that

$$
e^{-j \phi} x(n)+e^{j \phi} x(n-p)+\sum_{k=1}^{p} g_{k} x(n-k)=0
$$

Equ. (4) denotes some special form of prediction where using $x(n-1), \cdots, x(n-p+1)$ we predict in the forward and the backward direction a combination of $x(n)$ and $x(n-p)$. It is exactly this predictor we are going to call the Complex Bidirectional Predictor (CBP) which constitutes a generalization of the Bidirectional Predictor introduced in [4] for the real case.

Our goal is, using the available data samples, to estimate a CBP of order $M$ and then by rooting the corresponding polynomial to estimate the frequencies $f_{k}$. We must select $M \geq p$ in order to be able to identify correctly all frequencies (actually we must select $M \gg p$ in order to have a good estimation). Thus if $\boldsymbol{g}_{M+1}=$

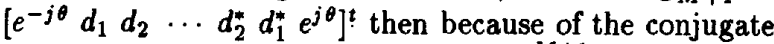
symmetry property we must estimate $\left\lceil\frac{M+1}{2}\right\rceil$ parameters. Notice that the parameter $\theta$ is of a special form and thus 
we need to distinguish it from the other parameters $d_{k}$, thus let

$$
\boldsymbol{g}_{M+1}=\left[e^{-j \theta} d_{M-1}^{t} e^{j \theta}\right]^{t}
$$

where $d_{M-1}$ is again a conjugate symmetric vector. If we now use least squares to estimate $\theta$ and $\boldsymbol{d}_{M-1}$ then we can easily show that we must minimize the following cost function with respect to $\theta$ and $d_{M-1}$

$$
\mathcal{E}\left(\boldsymbol{d}_{M-1}, \theta\right)=\boldsymbol{g}_{M+1}^{H} Q_{M+1} \boldsymbol{g}_{M+1}
$$

where the matrix $Q_{M+1}$ is the associated data autocorrelation matrix defined as

$$
\begin{aligned}
& Q_{M+1}=R_{M+1}+J R_{M+1}^{*} J \\
& R_{M+1}=\sum_{n=M+1}^{N} x_{M+1}^{*}(n) x_{M+1}^{t}(n)
\end{aligned}
$$

where $x_{M+1}(n)=[x(n) \cdots x(n-M)]^{t}$. The matrix $Q_{M+1}$ is positive definite, Hermitian and Persymmetric, that is $Q_{M+1}=Q_{M+1}^{H}$ and $Q_{M+1}=J Q_{M+1}^{*} J$. Let us define a partition of $Q_{M+1}$ that is necessary for our problem

$$
Q_{M+1}=\left[\begin{array}{ccc}
q & \boldsymbol{q}_{M-1}^{H} & s \\
\boldsymbol{q}_{M^{* 1}} & Q_{M-1} & J \boldsymbol{q}_{M-1}^{*} \\
s^{*} & \boldsymbol{q}_{M-1}^{t} J & q
\end{array}\right]
$$

To obtain the optimum parameters we shall perform the minimization of Equ. (6) in two consecutive steps, first we shall minimize $\mathcal{E}\left(\boldsymbol{d}_{\boldsymbol{M - 1}}, \theta\right)$ with respect to $\boldsymbol{d}_{\boldsymbol{M}-1}$ assuming $\theta$ given and then the resulting expression with respect to $\theta$. One can easily show that

$$
\mathcal{E}(\theta)=\min _{\boldsymbol{d}_{M-1}} \mathcal{E}\left(\boldsymbol{d}_{M-1}, \theta\right)=2 \operatorname{Re}\left\{a_{M+1} e^{j \theta}\right\}
$$

where $a_{M+1}$ and the optimum vector $\boldsymbol{d}_{M-1}$ are defined by

$$
\begin{aligned}
& \boldsymbol{d}_{M-1}=-\left(J \boldsymbol{p}_{M-1}^{*} e^{-j \theta}+\boldsymbol{p}_{M-1} e^{j \theta}\right) \\
& \boldsymbol{p}_{M-1}=-Q_{M-1}^{-1} J \boldsymbol{q}_{M-1}^{*} \\
& a_{M+1}=q e^{-j \theta}+s e^{j \theta}+\boldsymbol{q}_{M-1}^{H} \boldsymbol{d}_{M-1}
\end{aligned}
$$

Minimizing now $\mathcal{E}(\theta)$ with respect to $\theta$, after some algebra it can be shown that the optimum $\theta$ is given by

$$
\theta=0.5\left(\pi-\arg \left\{s+q_{M-1}^{H} p_{M-1}\right\}\right)
$$

Eqs. $(10,11)$ constitute the complete solution to the estimation problem. If we are though interested in solving the problem efficiently it is possible to derive an order recursive algorithm. Notice that all optimum quantities can be written in terms of the auxiliary vector $p_{M-1}$. This vector can be easily obtained as a by-product of the order recursive modified covariance algorithm of complexity $O\left(M^{2}\right)$ presented in [1].

Until now the only well known symmetric predictor applicable to the problem of spectral line estimation is the Complex Smoother Predictor (CSP), which estimates in the Least Squares sense the sample $x(n)$ using $m$ past and $m$ future samples. The CSP is a conjugate symmetric predictor of only odd length that has the form

$$
s_{2 m+1}=\left[\begin{array}{lll}
\boldsymbol{b}_{m}^{t} & 1 & \boldsymbol{b}_{m}^{H}
\end{array}\right]^{t}
$$

The optimum CSP is estimated using a similar as the CBP Least Squares problem, namely the predictor that minimizes

$$
\mathcal{E}\left(s_{2 m+1}\right)=s_{2 m+1}^{H} Q_{2 m+1} s_{2 m+1}
$$

The optimum predictor is found by solving the following equation

$$
Q_{2 m+1} s_{2 m+1}=\left[\begin{array}{lll}
0_{m}^{t} & \rho & 0_{m}^{t}
\end{array}\right]^{t}
$$

An interesting fact is that the CSP can also be derived as a by-product of the same order recursive algorithm used for the CBP [1].

The estimation of the frequencies (which is our final goal) is achieved by rooting the polynomials formed by the predictors (CBP or CSP).

\section{ASYMPTOTIC PERFORMANCE OF CBP AND CSP}

It is very difficult in general to compute a performance measure for the two frequency estimators for the finite data case. In order to be able to derive some semianalytic results we shall consider the asymptotic (infnite data number) case. For the asymptotic case we shall show some very important properties for both predictors. These properties seem to hold for the finite data case as it is confirmed by a large number of experiments.

A. Asymptotic Bias.

Both predictors are biased even for the asymptotic case. Actually this is a fact for all known linear LS predictors applied to the same problem. Since the CSP can only be defined for odd orders, we shall compute the bias for this case only. As we have seen in the previous section the two predictors are defined through similar optimization problems. Let us see what is the form of these problems in the asymptotic case. Notice that the optimum estimated quantities remain the same if instead of the matrix $Q$ we use the normalized matrix $Q / N$, where $N$ is the number of available data points. Letting $N \rightarrow \infty$ the autocorrelation matrix takes the form

$$
Q_{2 m+1}=\sigma_{w}^{2} I+V_{2 m+1}^{H} V_{2 m+1}
$$

where $\sigma_{w}^{2}$ is the noise variance and

$$
\begin{aligned}
& V_{2 m+1}=\left[\boldsymbol{v}_{2 m+1}^{1} \cdots \boldsymbol{v}_{2 m+1}^{p}\right] \\
& \boldsymbol{v}_{2 m+1}^{k}=\left[e^{-j m 2 \pi f_{k}} \cdots 1 \cdots e^{j m 2 \pi f_{k}}\right]^{t}
\end{aligned}
$$

We also have assumed that all complex exponentials that constitute the signal $x(n)$ have unit amplitude. To find the optimum predictors for the asymptotic case we follow exactly the same steps as in the finite data case, that is Eqs. $(10,11)$ for the CBP and Equ. (14) for the CSP. The obtained predictors are functions of the frequencies $f_{k}, k=1, \ldots, p$. Using these formulae for the predictors we can form the corresponding polynomials and thus by rooting them obtain estimates of the frequencies for both predictors. Unfortunately it is not possible to derive closed form solutions for the estimated frequencies except for very small orders $m$. In any case this is not so important since we can always find numerically for an adequate number of frequency combinations the asymptotic estimates and compare the two methods. For example in the 
FIGURE 1

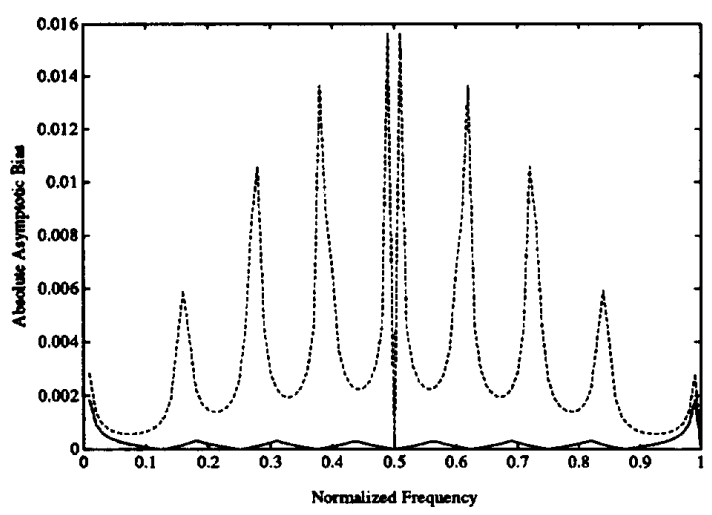

case of a process consisting of two exponentials in noise a 3-D plot is required to illustrate the performance of a method. However it can be shown that the asymptotic performance of both methods depend only on the difference $\left|\Delta f=f_{1}-f_{2}\right|$ and the estimation problem of the two frequencies is equivalent to the estimation of $\Delta f$. Thus in Figure 1 we plot the estimation error $|\widehat{\Delta f}-\Delta f|$ versus $\Delta f$ for the two predictors. The solid line corresponds to the CBP and the dashed to the CSP. We used $m=7$ for both cases and $\sigma_{w}^{2}=0.01$. Clearly the CBP exhibits a much smaller bias with respect to the CSP. The results for other orders and other values of the noise variance are similar.

\section{B. Location of the Roots.}

A very important problem in the estimation of the undamped exponentials through the use of predictors is the determination of the roots of the corresponding polynomials. We shall show that in the case of the CBP for the asymptotic case the roots are situated on the unit circle. Notice first that the asymptotic matrix $Q_{M+1}$ is Toeplitz and also positive definite because of the existence of the term $\sigma_{w}^{2} I$. If we use the partitioning of Equ. (8) and define the forward predictor $\left[\begin{array}{ll}1 & a_{M-1}^{t}\end{array}\right]^{t}$ that solves the following Wiener problem

$$
\left[\begin{array}{cc}
q & q_{M-1}^{H} \\
q_{M-1} & Q_{M-1}
\end{array}\right]\left[\begin{array}{c}
1 \\
a_{M-1}
\end{array}\right]=\left[\begin{array}{c}
r_{x} \\
0_{M-1}
\end{array}\right]
$$

which is known from the Levinson-Durbin algorithm that leads to a stable predictor [2]. If we define

$$
\boldsymbol{g}_{\boldsymbol{M}+1}=e^{-j \theta}\left[\begin{array}{lll}
1 & \boldsymbol{a}_{M-1}^{t} & 0
\end{array}\right]^{t}+e^{j \theta}\left[\begin{array}{lll}
0 \boldsymbol{a}_{M-1}^{H} & 1
\end{array}\right]^{t}
$$

then this predictor is the optimum CBP. This statement can be proved by showing that the predictor satisfies the equations that define the optimum CBP $(10,11)$. Since our predictor can be written under the form of Equ. (18) from [8] we have that it will have all its roots on the unit circle. This property does not hold for the CSP as one can verify by a counterexample (take for instance the case $M=2$ that can be solved analytically). Even though the result is asymptotic, extensive simulations have shown that it is still valid in the finite data case, provided that the matrix $Q$ is well conditioned. Due to this property of the CBP a significant computational saving is achieved in the rooting procedure, since we can use algorithms that search zeros only on the unit circle (e.g. [7]).

C. Asymptotic Variance.

A very important performance measure is the estimation error variance. Analytic expressions for this case are very difficult even for the asymptotic case. However since this measure is very important we shall investigate this matter in the next section through extensive simulations, as it is usually the case in the literature.

\section{COMPARISON OF LS SYMMETRIC PREDICTORS}

So far we have shown that the CBP exhibits less asymptotic bias than the CSP and that its roots lie on the unit circle implying a simpler rooting procedure.

To confirm these results for the finite data case and also study the variance of the frequency estimates for both symmetric predictors we conducted the typical experiment of [5]. A total of 500 independent data blocks, with 25 data points each, were generated using the formula

$$
x(n)=e^{j 2 \pi 0.5 n}+e^{j(2 \pi 0.52 n+\pi / 4)}+w(n)
$$

for $n=1, \ldots, 12500$, where $w(n)$ is a complex white noise with variance $\sigma_{w}^{2}$. The SNR per exponential is defined as $10 \log _{10}\left(1 / 2 \sigma_{w}^{2}\right)$ and SNR values range from $10 \mathrm{db}$ to $30 \mathrm{db}$. For each block of data, each SNR value and each estimation method, as frequency estimates were taken the angles of the roots that were closest to the true frequencies. This is an ideal case of course but it is indicative of the true performance of each method. In Figure 2 the variance $\sigma_{f}^{2}$ of the frequency estimate of $f_{1}$ is plotted in logarithmic scale vs SNR, for the different methods. For each particular method the order that had the best performance was selected. The top solid line depicts the Cramer-Rao bound. The dashed line corresponds to the CBP with order equal to 12. The bottom solid line corresponds to the CSP of order 13 and the dotted line corresponds to the Forward-Backward Linear Prediction (FBLP) method with order 13. The performance of the CBP is slightly inferior to that of FBLP while the CSP has a significantly poorer performance. Finally the dashed-dotted line corresponds to a combination of the CBP and CSP which unfortunately due to the limited space shall not be discussed here. Notice that the combination is superior to the performance of the FBLP and can be achieved with negligible added complexity.

\section{SVD BASED ESTIMATION FOR CBP AND CSP}

In this section a minimum norm method, similar to the one in [6], will be presented for obtaining symmetric predictors. In all these methods there is the need of performing SVD to a data correlation matrix in order to reduce the effects of the noise. In our case this role is played by the matrix $Q_{M+1}$. Because of the symmetry properties of this matrix the singular vectors can be conjugate symmetric. The collection of singular vectors can be divided into two parts, one spanning the signal subspace and the other spanning the noise subspace. The signal subspace has dimension $p$ and the noise subspace $M+1-p$. The problem is to find a conjugate symmetric vector of a specific form 


\section{FIGURE 2}

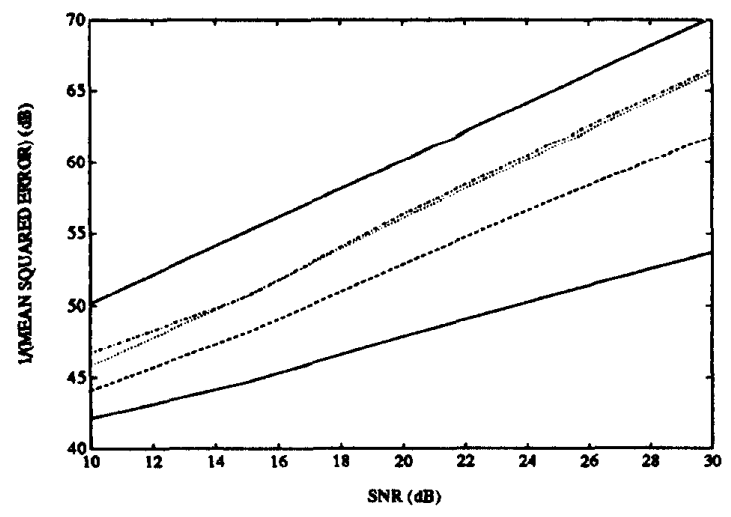

(CBP or CSP) that is orthogonal to the signal subspace and has minimum norm. Thus the vector we are looking for belongs to the noise subspace and in consequence it is a linear combination of the corresponding singular vectors. Since these vectors are conjugate symmetric, in order the resulting vector to be conjugate symmetric as well, we must use real coefficients for the combination. If we consider first the CBP $g_{M+1}$ then

$$
g_{M+1}=V_{n} c_{M+1-p}
$$

where $V_{n}$ is a matrix that has as columns the $M+1-$ $p$ singular vectors of the noise subspace and $c_{M+1-p}$ is a real vector. Let now $u_{M+1-p}$ denote the top row of the matrix $V_{n}$ then because of the orthonormality of the singular vectors we have $\left\|g_{M+1}\right\|=\left\|c_{M+1-p}\right\|$ and thus the problem we need to solve is equivalent to

$$
\begin{aligned}
& \min _{c}\left\|c_{M+1-p}\right\| \text { subject to } \\
& \left|\boldsymbol{u}_{M+1-p}^{t} c_{M+1-p}\right|=1
\end{aligned}
$$

The constraint in (21) expresses the requirement that the first element of $g_{M+1}$ is of unit modulus (see Equ. (5)). For the CSP the corresponding problem is exactly similar, the only difference being the vector $u$ which now is the central row of the matrix $V_{n}$ expressing the requirement that the central element of $s_{2 m+1}$ is unity (see Equ. (12)). If $u=u_{R}+j u_{I}$ it can be proved that the solution in the CBP case is the eigenvector corresponding to the largest eigenvalue of the rank-2 matrix $u_{R} u_{R}^{t}+u_{I} u_{I}^{t}$ and in the CSP case $c=u$.

Significant saving in the SVD process can be achieved by utilizing the symmetries of the matrix $Q_{M+1}$. Specifically the SVD problem can be reduced to the SVD of the following real symmetric matrix

$$
L_{M+1}=\left[\begin{array}{cc}
A_{R}+B_{R} J & -A_{I}+B_{I} J \\
-A_{I}^{t}+J B_{I}^{t} & A_{R}-B_{R} J
\end{array}\right]
$$

where $A_{R}+j A_{I}$ is the upper left quadrant of the matrix $Q_{M+1}$ and $B_{R}+j B_{I}$ is the upper right quadrant of the same matrix. If $\left[\boldsymbol{y}_{R}^{t} \boldsymbol{y}_{I}^{t}\right]^{t}$ is an eigenvector of the matrix in (22) then the corresponding eigenvector of $Q_{M+1}$ is $\left[y^{t} y^{H} J\right]^{t}$ where $y=y_{R}+j y_{I}$.
Simulation Results.

To test the performance of the presented SVD methods we conducted the same experiment as in Section IV. The results are presented in Figure 3 . The top solid line is again the Cramer-Rao bound. The dashed line shows the performance of the CBP with order 16 , the bottom solid line is the CSP method with order 16 and the dotted line is the FBLP method with order 18 . Finally the dash-dotted line is the combination of the CBP and the CSP. Notice that the CBP performs almost identically with the FBLP while the combination CBP and CSP performs slightly better. Also extensive simulations have shown that the roots of the CBP polynomial lie on the unit circle leading again to fast rooting algorithms.

\section{FIGURE 3}

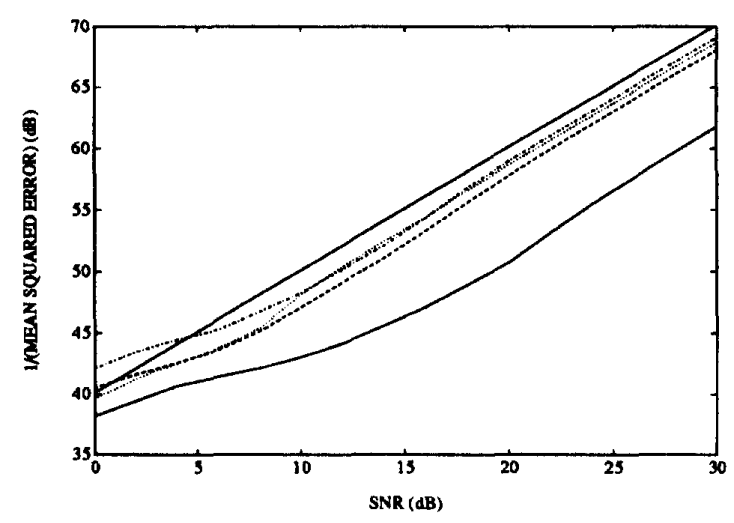

\section{References}

[1] S.L. Marple, "Digital Spectral Analysis with Applications", Prentice Hall, New Jersey, 1987.

[2] S.M. Kay, "Modern Spectral Estimation: Theory and Applications", Prentice Hall, New Jersey, 1988.

[3] M. Amin and L. J. Griffiths, "Time Varying Spectral Estimation Using Symmetric Smoothing", Proc. of the ICASSP-83, pp. 9-12, Boston, 1983.

[4] K. Berberidis and S. Theodoridis, "New Levinson, Schur and Lattice Type Algorithms for Linear Phase Filtering", IEEE Trans. on ASSP, Vol. 38, No. 11, pp. 1879-1892, Nov. 1990.

[5] D.W. Tufts and R. Kumaresan, "Estimation of Frequencies of Multiple Sinusoids: Making Linear Prediction Perform Like Maximum Likelihood", Proc. of IEEE, Vol. 70, No. 9, pp. 975-989, Sept. 1982.

[6] R. Kumaresan and D.W. Tufts, "Estimating the Angles of Arrival of Multiple Plane Waves", IEEE Trans. on Aerosp. Electon. Syst., Vol. 19, pp. 134-139, June 1983.

[7] W.S. McCormick and J.L. Lansford, "Efficient Parallel Rooting of Complex Polynomials on the Unit Circle", IEEE Trans. on SP, Vol. 39, No. 10, pp. 2347-2351, Oct. 1991.

[8] D.M. Goodman and E.K. Miller, "A Note on Minimizing the Prediction Error when the Zeros are Restricted to the Unit Circle", IEEE Trans. on ASSP, Vol. 30, No. 3, pp. 503-505, June 1982 . 